\title{
Die Kosten unseres Gesundheitswesens
}

Johannes Ledergerber

Korrespondenz:

Dr. med. Johannes Ledergerber

Facharzt für Kinder

und Jugendliche FMH

Badenerstrasse 681

CH-8048 Zürich

j.d.ledergerber@hin.ch
Mit Interesse habe ich Ihren Artikel über «Unser Gesundheitswesen im internationalen Vergleich» in der Ausgabe Nr. 44 der SÄZ gelesen [1]. Zu Beginn war ich beeindruckt von der schieren Menge von 600000 Einzeldaten. Im Lauf der Lektüre begann ich mich aber darüber zu wundern, wie wenig Relevantes über das Gesundheitswesen sich aus diesen Daten offensichtlich ablesen lässt. Und schliesslich wunderte ich mich über Titel und Zwischentitel, die solche Relevanz suggerieren. In Ihrem Artikel geht es lediglich um die Kosten unseres Gesundheitswesens, nicht um das Gesundheitswesen als Ganzes, wie der Titel suggeriert. Standardisierung auf nationalökonomische Kennziffern ist ein gängiges Verfahren, aber ein echter Kostenvergleich macht eigentlich nur Sinn, wenn die Kosten an den Zielen der Gesundheitspolitik gemessen werden.

Wie Sie richtig bemerken, sind die Gesundheitskosten von äusserst vielen Faktoren abhängig. Und ebenso richtig ist vermutlich auch die Feststellung, dass die Gewichtung der einzelnen Faktoren schwierig ist und man nicht den Faktor per se findet, der in erster Linie kostentreibend ist. Und trotzdem sind mir beim Lesen ein paar Gedanken durch den Kopf gegangen, die ich Ihnen gerne mitteilen möchte.

1. Ich kann mir vorstellen, dass in einigen anderen OECD-Ländern die Personalkosten wesentlich geringer sind als bei uns. Ich meine damit aber ganz entschieden nicht, dass sie bei uns im Vergleich zum übrigen Lebensstandard zu hoch sind, sondern im Gegenteil, dass in den anderen OECD-Ländern sich sowohl Ärzte als auch medizinisches Pflege- und Hilfspersonal oft mit Hungerlöhnen abfinden müssen. Selbst in England sind viele froh, wenn sie überhaupt einen Job haben. Wenn dort die Leute anständig besoldet würden, sähe die Statistik wahrscheinlich anders aus. Das soll jetzt nicht eine Einladung sein, unser Personal schlechter zu bezahlen, denn der Beruf als Pflegefachfrau oder -mann ist physisch und psychisch äusserst belastend und ist selbst in der Schweiz im Vergleich zu anderen Jobs (z. B. bei einer Versicherung oder Bank) zumeist anspruchsvoller und dabei knapp angemessen bezahlt.

2. Die Alterspflege in der Schweiz ist nicht nur wegen der demographischen Trends teurer, sondern vermutlich auch, weil die alten Leute hier einerseits häufiger aus dem Familienkreis abgeschoben werden als in lateinischen, osteuropäischen, skandinavischen und anderen Ländern (ausser in Deutschland), und weil man andererseits kaum Personal findet, das bei einer allfälligen Pflege zu Hause zu einem noch verkraftbaren Lohn mithilft.

3. Sie sagen zu Recht, dass die Effektivität der Gesundheitssysteme nicht in erster Linie von der Höhe der Gesundheitsausgaben abhängt, sondern von vielen anderen Faktoren. Und doch ist es auf der anderen Seite so, dass wir in der Schweiz einen sehr hohen, flächendeckenden und noch für alle zugänglichen Standard haben, der sich international zeigen lässt. Wenn ich in England für eine Hüftprothese an öffentlichen Spitälern 1-2 Jahre warten muss, dann darf das auch nicht so viel kosten. Lebensqualität hat halt doch ihren Preis. Ohne grossen finanziellen Aufwand kann man in der Medizin heute keine Topstandards erreichen. Allerdings ist viel Geld nur eine notwendige, keine hinreichende Bedingung für Topmedizin. Dahinter stecken immer Menschen, die sich mit Herzblut für ihre Sache einsetzen, wie dies in der Schweiz die meisten Allgemeinpraktiker, das Pflegepersonal und viele Spezialisten tun.

4. Ärztedichte: In der Schweiz ist wohl der Frauenanteil im Vergleich zu den anderen OECD-Ländern überdurchschnittlich hoch. Ein sehr grosser Anteil der Ärztinnen arbeitet Teilzeit, und zwar um die 20-40\% (nicht etwa 70-80\%). Das führt statistisch zu einer höheren Ärztedichte, aber nicht zu einer dichteren Versorgung und daher auch nicht zu höheren Kosten. Und weil dem so ist, hat ein Ärztestopp höchstens populistische Relevanz. Ich teile Ihr Bedauern darüber, dass nur wenige Daten über Mediziner und Arztpraxen vorliegen bzw. diese Daten nicht zugänglich sind. Auch deshalb sollte man nicht über eine überdurchschnittliche Ärztedichte sprechen und schon gar nicht in einem Untertitel.

Ich finde es richtig, dass sich alle Beteiligten bemühen, die Kosten so weit es geht in den Griff zu bekommen oder - so könnte man auch sagen im Griff zu halten. Auf der anderen Seite habe 
ich aber auch den Eindruck, dass man sich heute mehr und mehr daran gewöhnt hat, alles immer billiger zu bekommen, und dabei schweigend in Kauf nimmt, dass sich andernorts für uns Millionen von Menschen zu einem Hungerlohn abrackern. Die Medizin ist teurer geworden, teurer als die anderen Dienstleistungen in der Schweiz, ausgenommen das Bankenwesen (65 Milliarden lassen grüssen), und viel teurer als die Produkte aus den Billiglohnländern.

Das hat zwei offensichtliche Gründe: Zum einen liegt es daran, dass wir unsere Gesundheit eben nicht wie unsere Kleider und Laptops in Niedriglohnländern herstellen lassen können. Seit meiner Kindheit sind in der Schweiz die Löhne gerechter geworden. Damals haben zwei Nonnen pro Spitalstockwerk für Gotteslohn die Patienten in den Spitälern mit ihrem ganzen Lebenseinsatz gepflegt - Tag und Nacht. Heute arbeitet pro Stockwerk ein Vielfaches an Pflegefachpersonal, weil die Pflege viel komplizierter, die Patienten wesentlich anspruchsvoller und die Arbeitszeiten angemessener wurden. Zum andern sind die steigenden Kosten darauf zurückzuführen, dass sich die Medizin und damit das Gesundheitswesen in den letzten 40 Jahren um ein Vielfaches verbessert hat und sich ständig weiter perfektioniert. Noch vor 40 Jahren war es keine Besonderheit, selbst als junger Mensch im Spital zu sterben. Heute ist das die grosse Ausnahme. Ein Tumor war damals meistens ein sicheres Todesurteil, Poliomyelitis eine Krankheit, deren Folgen in der Schule und im Alltag sichtbar waren, eine kongenitale Hüftluxation ein sicherer Weg in die Invalidität; man könnte diese Liste beliebig verlängern. Warum spricht man heute so wenig davon?

Ich mache mir grosse Sorgen um die Zukunft unserer flächendeckenden ärztlichen Grundversorgung, die in erster Linie durch die Allgemeinpraktiker und die Pädiater gewährleistet wird. Heute hat man sich daran gewöhnt, über die Kosten des Gesundheitswesens aus einer rein ökonomischen Perspektive zu urteilen, ohne die Kosten an den demokratisch vereinbarten gesundheitspolitischen Zielsetzungen und den sich daraus ergebenden medizinischen Leistungen zu messen. Es liegt in unserer Verantwortung, hier in die Diskussion einzugreifen. Wir dürfen unser Gesundheitswesen nicht dauernd unter Beschuss nehmen und uns am Schluss wundern, wenn wir vor einem Scherbenhaufen stehen.

\section{Literatur}

1 Kocher G. Unser Gesundheitswesen im internationalen Vergleich. Schweiz Ärztezeitung. 2008;89(44):1911-6. 